\title{
Non-invasive Derivation of 3D Systolic Nonlinear Wall Stress in a Biventricular Model from Tagged MRI
}

\author{
Aichi Chien ${ }^{1}$, J. Paul Finn ${ }^{2}$, and Carlo D. Montemagno ${ }^{1}$ \\ ${ }^{1}$ Dept. of Biomedical Engineering, University of California Los Angeles, CA 90095, USA \\ aichi@seas . ucla.edu \\ ${ }^{2}$ Dept. of Radiological Sciences, University of California, Los Angeles, CA 90095, USA
}

\begin{abstract}
We present a nonlinear finite element method to calculate the local myocardial wall stress in a reconstructed biventricular MRI model. Nonlinear formulations are utilized in order to describe the ventricular large deformation. Using incremental force computation, a dynamic model showing the change of myocardium wall stress during systolic contraction was established. The preliminary results show that in the normal human heart the local stress increases by a factor of $10^{4}$ from the end of diastole to the end of systole. Furthermore, during the systolic process the left ventricle develops three times more inner wall stress than the right ventricle, and the peak inner wall stress areas in both ventricles are located at the apex.
\end{abstract}

\section{Introduction}

To gain insight into the mechanisms of heart failure, it is necessary to elucidate the dynamic forces generated by the ventricles. Abnormal heart wall stress (WS) is known to be an important factor leading to cardiac dysfunction. Evidence for this is that regional ventricular remodeling is closely related to ventricular stress [1]. The goal of this paper is to present a nonlinear finite element (FE) model based on tagged MRI to calculate the 3D WS and provide information necessary to better understand the mechanisms of cardiac dysfunction. We believe this is the first attempt to solve human biventricular WS using a nonlinear large deformation formulation in a gross model. The benefit of this method is that it avoids the breakdown of linear theory which usually happens when the strain is larger than $10 \%$ - the type of deformation typical in cardiac contraction [2].

\section{Method and Results}

A 3D biventricular gross model representing normal heart geometry was constructed based on published MRI data [2]. Then, using the FE program COSMOS, we divided the gross model into 29,136 tetrahedral mesh elements. The formulations included nonlinear myocardium material properties with large deformation equations [3]. Furthermore, we defined five time intervals in a $450 \mathrm{msec}$ contraction process and as- 
signed incremental force on these intervals. The force representing blood resistance and the torsion/contraction force were both considered in our simulation. The total number of equations was $1,359,105$ and the overall calculation time was $24,998 \mathrm{sec}$.

The ventricular local stress changes in systole show that the stress increases as the ventricles contract (Fig.1). The WS, on average, increases by a factor of $10^{4}$ during the contraction. A nonuniform stress distribution is observed which suggests pumping efficiency may drop if the muscle in this high stress area develops defects. The calculation also indicates that the left ventricle (LV) has three times higher inner WS compared to the right ventricle (RV) and that the maximum local stress of $1.6356 \times 10^{5}$ $\mathrm{Pa}$ occurs in the apex of the LV. This high stress area is more likely to change the myocardium elasticity and may explain that infarction expansion tends to occur more frequently among patients with infractions involving the apex of the ventricles [4]. Comparing the calculated inner and outer ventricular WS, the results show that inner ventricular WS increases more during the contraction. It may suggest that myocardium contractive force is mainly produced by the inner region of the ventricular wall.

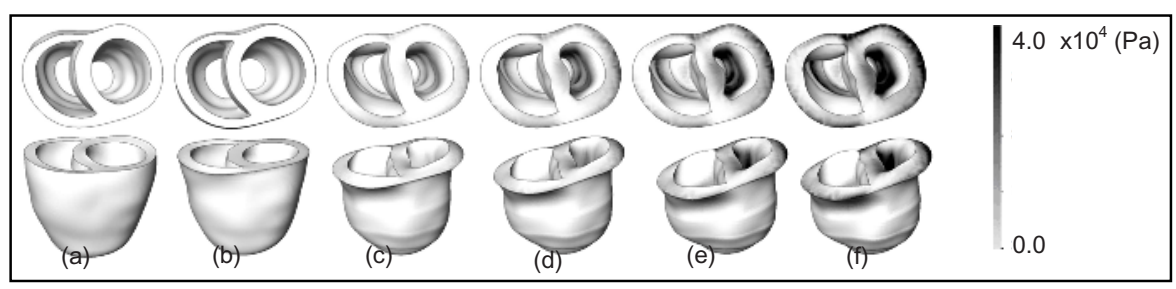

Fig. 1. 3D stress distribution on ventricles from the end of diastole (a) to the end of systole (f).

\section{Conclusion}

The model presented here uses 3D MRI to reconstruct the ventricular geometry, coupled with nonlinear FE methods. Preliminary results show the stress distribution in the normal heart contraction, providing unique insight into cardiac function in an entirely non-invasive way. However, a more accurate stress calculation will require correct data for the material properties of the myocardium in-vivo. Further material formulation and clinical model validation are currently in progress.

\section{References}

1. Grossman, W., Jones, D. \& McLaurin, L.P. Wall stress and patterns of hypertrophy in the human left ventricle. J Clin Invest 56, 56-64 (1975).

2. Haber, I., Metaxas, D.N. \& Axel, L. Three-dimensional motion reconstruction and analysis of the right ventricle using tagged MRI. Med Image Anal 4, 335-55 (2000).

3. Hunter, P.J., McCulloch, A.D. \& ter Keurs, H.E. Modelling the mechanical properties of cardiac muscle. Prog Biophys Mol Biol 69, 289-331 (1998).

4. Pfeffer, M.A. Left ventricular remodeling after acute myocardial infarction. Annu Rev Med 46, 455-66 (1995). 